\title{
CAPÍTULO 08: TECNOLOGIAS DIGITAIS NO ENSINO SUPERIOR: DESENVOLVIMENTO DE UM APLICATIVO PARA O ESTUDO DA AGRONOMIA
}

\section{CAPÍTULO 08: TECNOLOGÍAS DIGITALES EN EDUCACIÓN SUPERIOR: DESARROLLO DE UNA APLICACIÓN PARA EL ESTUDIO DE LA AGRONOMÍA}

\author{
CHAPTER 08: DIGITAL TECHNOLOGIES IN HIGHER EDUCATION: \\ DEVELOPMENT OF AN APPLICATION FOR THE STUDY OF AGRONOMY
}

Bruno Argente de Sousa ${ }^{1}$; Lailson da Conceição de Brito ${ }^{2}$; Gabriel Schardong Ferrão ${ }^{3}$; Claudinéia Aparecida Queli Geraldi ${ }^{4}$; Sumaya Ferreira Guedes ${ }^{5}$

DOI: https://doi.org/10.31692/978-65-88970-17-1.92-106

\section{RESUMO}

Com a pandemia em 2020, o uso das tecnologias se tornou uma das possibilidades para o andamento das aulas não presenciais, seja no ensino básico ou superior. Dessa forma, proporcionou aos educadores o repensar sobre as práticas de ensino adotadas com a respectiva inclusão de tecnologias digitais nas aulas. Nesse sentido, este trabalho teve como objetivo o desenvolvimento de um jogo, em formato de quiz, para aparelho móvel com conteúdos relacionados a Agronomia para estudantes no primeiro semestre. Inicialmente foi realizada uma pesquisa nos projetos pedagógicos do curso de Agronomia da UNEMAT, campus de Nova Mutum para avaliar as disciplinas ofertadas nos primeiros semestres. Por ser um protótipo, foi selecionado inicialmente duas disciplinas com conteúdo geral (Física e Bioquímica) e três específicas do curso de Agronomia (Natureza e Propriedade do Solo, Agrometeorologia e Comunicação e Extensão Rural). Após a seleção, foram elaboradas cinco questões de cada disciplina e elaborado um texto simplificado com a explicação do conteúdo, para que os alunos usuários possam estudar os conteúdos ali propostos. Na programação do aplicativo, na tela inicial o usuário deverá selecionar o nome e o tipo do avatar do seu agro-estudante. Inicialmente apenas uma opção de roupa será disponibilizada, sendo liberada outras conforme os acertos nas questões. Sempre que o usuário acertar a questão, aparecerá na tela uma mensagem de Parabéns! Se a resposta marcada for errada, a mensagem será de continue tentando. Cada questão (subfases) terá inicialmente 50 pontos. Se o aluno errar uma vez, ela passará a valer 40 pontos até que tenha valor de 10 pontos quando esgotar as alternativas, ou seja, o aluno perde 10 pontos a cada resposta errada que marcar. No final do jogo, o aluno poderá acumular um valor máximo de 1250 pontos ou um valor mínimo de 250 pontos. É importante destacar que a cada finalização das fases (disciplinas), o usuário terá acesso a outras roupas do avatar. Se errar a questão, o aluno será direcionado para a tela do conteúdo para estudo, podendo retornar para uma nova tentativa. No menu inicial o aluno também poderá acessar os conteúdos para estudo, podendo estudar antes de iniciar o jogo. Com o aplicativo, o aluno ingressante poderá utilizar para estudo e dessa forma, auxiliar na fixação do aprendizado.

Palavras-Chave: Plataforma Android; Jogos didáticos; Tecnologias Digitais; Objetos digitais de aprendizagem.

\begin{abstract}
RESUMEN
Con la pandemia en 2020, el uso de tecnologías se ha convertido en una de las posibilidades para el progreso de las clases no presenciales, ya sea en la educación básica o superior. De esta manera, brindó a los educadores un replanteamiento de las prácticas docentes adoptadas con la respectiva inclusión de las tecnologías digitales en las clases. En este sentido, este trabajo tuvo como objetivo desarrollar un juego, en formato de cuestionario, para dispositivo móvil con contenidos relacionados con la Agronomía para estudiantes del primer semestre. Inicialmente, se realizó una investigación en los Proyectos
\end{abstract}

\footnotetext{
${ }^{1}$ Agronomia, Universidade do Estado de Mato Grosso, brunoargente@ outlook.com

${ }^{2}$ Agronomia, Universidade do Estado de Mato Grosso, lailsonbrito173@gmail.com

${ }^{3}$ Ciências da Computação, Universidade do Estado de Mato Grosso, gabrielferrao@ unemat.br

${ }^{4}$ Agronomia, Universidade do Estado de Mato Grosso, claudineia.geraldi@ unemat.br

${ }^{5}$ Agronomia, Universidade do Estado de Mato Grosso, sumayaguedes@ unemat.br
} 
Pedagógicos del Curso de Agronomía en Unemat, campus Nova Mutum para evaluar las asignaturas ofrecidas en los primeros semestres. Por tratarse de un prototipo, inicialmente se seleccionaron dos asignaturas de contenido general (física y bioquímica) y tres específicas del curso de Agronomía (Naturaleza y Propiedad del Suelo, Agrometeorología y Comunicación y Extensión Rural). Tras la selección, se elaboraron cinco preguntas de cada disciplina y se elaboró un texto simplificado con la explicación del contenido, para que los alumnos usuarios puedan estudiar los contenidos allí propuestos. Al programar la aplicación, en la pantalla de inicio el usuario debe seleccionar el nombre y tipo de avatar de su Agro-alumno. Inicialmente, solo una opción de ropa estará disponible, otras se publicarán de acuerdo con la exactitud de las preguntas. Siempre que el usuario responda correctamente, aparecerá un mensaje de enhorabuena en la pantalla. Si la respuesta marcada es incorrecta, el mensaje seguirá intentándolo. Cada pregunta (subfases) tendrá inicialmente 50 puntos. Si el alumno se equivoca una vez, valdrá 40 puntos hasta que valga 10 puntos cuando se agoten las alternativas, es decir, el alumno pierde 10 puntos por cada respuesta incorrecta que puntúe. Al final del juego, el alumno puede acumular un máximo de 1250 puntos o un mínimo de 250 puntos. Es importante tener en cuenta que en cada finalización de las fases (disciplinas), el usuario tendrá acceso a otras prendas de avatar. Si la pregunta es incorrecta, el estudiante será dirigido a la pantalla de contenido para estudiar y puede regresar para un nuevo intento. En el menú inicial el alumno también podrá acceder a los contenidos a estudiar, pudiendo estudiar antes de iniciar el juego. Con la aplicación, el alumno entrante podrá utilizarla para estudiar y de esta forma, asistir en la fijación del aprendizaje.

Palabras Clave: Plataforma Android, Juegos educacionales, Tecnologías digitales, Objetos de aprendizaje digitales.

\begin{abstract}
With the pandemic in 2020, the use of technologies has become one of the possibilities for the progress of non-face-to-face classes, whether in basic or higher education. In this way, it provided educators with a rethink about the teaching practices adopted with the respective inclusion of digital technologies in classes. In this sense, this work aimed to develop a game, in a quiz format, for mobile device with contents related to Agronomy for students in the first semester. Initially, a research was carried out in the Pedagogical Projects of the Agronomy Course at Unemat, Nova Mutum campus to evaluate the subjects offered in the first semesters. Because it is a prototype, two subjects with general content (physics and biochemistry) and three specifics of the Agronomy course (Nature and Soil Ownership, Agrometeorology and Communication and Rural Extension) were initially selected. After the selection, five questions of each discipline were elaborated and a simplified text was elaborated with the explanation of the content, so that the student users can study the contents proposed there. When programming the application, on the home screen the user must select the name and type of avatar of his Agro-student. Initially only one clothing option will be made available, others will be released according to the correctness of the questions. Whenever the user gets the question right, a Congratulations message will appear on the screen! If the marked answer is wrong, the message will be keep trying. Each question (subphases) will initially have 50 points. If the student makes a mistake once, it will be worth 40 points until it is worth 10 points when the alternatives are exhausted, that is, the student loses 10 points for each wrong answer that he scores. At the end of the game, the student can accumulate a maximum of 1250 points or a minimum of 250 points. It is important to highlight that at each completion of the phases (disciplines), the user will have access to other avatar clothes. If the question is wrong, the student will be directed to the content screen for study, and may return for a new attempt. In the initial menu the student will also be able to access the contents for study, being able to study before starting the game. With the application, the incoming student will be able to use it for study and in this way, assist in the fixation of learning.
\end{abstract}

Keywords: Android platform; Educational games; Digital Technologies; Digital learning objects.

\title{
INTRODUÇÃO
}

As tecnologias digitais passaram a ocupar um espaço primordial na comunicação e na vida das pessoas, com influência direta no cenário econômico, humano e social, transformando 
as relações sociais com maior evidência na pandemia iniciada no ano de 2020 (CARDOSO et al., 2020; RIEDNER E PISCHETOLA, 2016).

A maior parte da população atualmente possui acesso a algum tipo de tecnologias digitais como acesso a internet em computadores ou celulares. Dessa forma, as escola e as universidades precisam adotar propostas pedagógicas que utilizem diferentes tecnologia, principalmente a tecnologia digital (MORAES, 2002), pois se manifestam no contexto escolar através das informações e conhecimentos adquiridos por meio das mídias (RIEDNER E PISCHETOLA, 2016).

Entretanto, o uso das tecnologias no ambito educacional exige mudanças sociais e culturais que valorizam o novo tipo de saber e exige conhecimento com domínio de novas habilidades relacionadas as tecnologias digitais (PISCHETOLA, 2016).

Diversos recursos tecnológicos podem apoiar o processo de ensino, favorecendo a aprendizagem dos alunos. Nesse sentido, as tecnologias interativas como uso de programas para celulares podem ser aplicadas na educação, aumentando a pluraridade da abordagem dos conteúdos, atendendo diferentes estilos de aprendizagem e favorecendo o desenvolvimento de novas competências e habilidades do aluno (CARDOSO et al., 2020). Assim, o uso de objetos digitais podem auxiliar no ensino, incluindo no nível superior.

Nesse sentido, este trabalho teve como objetivo o desenvolvimento de um jogo, em formato de quiz, para aparelho móvel com conteúdos relacionados a Agronomia para estudantes no primeiro semestre.

\section{FUNDAMENTAÇÃO TEÓRICA}

\section{Tecnologias Digitais}

Os meios tecnológicos informativos e comunicativos, englobam uma ampla interação entre homem e as tecnologias, na qual ocorrem mudanças significativas ao decorrer dos anos na sociedade atual. Essas novas formas de comunicação proporcionam uma maior facilidade de aprendizado (KENSKI, 2008).

A evolução digital abrange novas formas das pessoas se comunicarem, mas como consequência pode determinar de forma direta ou indiretamente o agir ou pensar das pessoas na sociedade (LEVY, 1999).

No ensino, a eficiência gerada pela implantação dos métodos tecnológicos, proporcionando uma melhor interação entre ensinar e aprender, ocasionando uma ampla troca de conhecimentos entre os professores e alunos não apenas na sala de aula, visando a busca 
pelo conhecimento (CAROLINO, 2007).

Cabe ressaltar que a existência das tecnologias nas escolas não garantirá melhoria no aprendizado se ela não for devidamente planejada e usada de forma adequada. Portanto, é importante relações estabelecidas entre esses três elos da educação: professor, alunos e tecnologias, sendo desenvolvidos de uma forma mais unificada contribuirá nos entendimentos desses novos conceitos de aprendizado (VALENTE, 1999).

Com a pandemia da Covid-19, o uso das tecnologias se tornou uma das possibilidades para o andamento das aulas não presenciais e, dessa forma, proporcionou aos professores repensar sobre as práticas de ensino adotadas e qual o papel das tecnologias no ensino. Nesse sentido, a aprendizagem expandiu fronteiras devido a acessibilidade das tecnologias digitais de comunicação e informação (KENSKI, 2010).

\section{Objetos digitais de aprendizagem}

Objetos digitais de aprendizagem (ODA) segundo Wiley, (2000, p. 7) é "qualquer recurso digital que pode ser reusado para apoiar a aprendizagem". Nesse seguimento, tem surgido defesas a favor do ODA indicando que o mesmo tem apresentado papel significativo na aprendizagem e tem conseguido despertar interesse em discentes, devido a maneira dinâmica e interativa de aprendizado.

O ODA deve possuir um planejamento, desenvolvimento e uma padronização. Portanto, o objeto de aprendizagem deve desafiar os alunos seja em relação a máquina ou até mesmo com outros alunos.

A expansão do uso de objetos digitais de estudo, permitiu a educação de modo remoto. (ROVAI; JORDAN, 2004). Devido a pandemia causada pelo covid-19, as instituições de ensino, intensificaram o uso de objetos de estudo digitais contribuindo assim para uma fomação digital.

A utilização do ensino remoto pode proporcionar e restabelecer a iteração e troca de conhecimentos na sociedade acadêmica em um contexto onde a iteração presencial não pode continuar, e se tornou a opção mais eficaz a ser utilizada por vários países (ROVAI E JORDAN, 2004)

Ressalta-se também o fato dos meios de aprendizados terem sofrido mudanças significativas ao decorrer do século passado na sua formar de ensinar, contribuindo para uma nova reformulação nesse sistema na qual passou a ser voltada exclusivamente a quem aprende (TEODORO; VASCONCELOS, 2005). 
O uso dos recursos digitais na educação alicerça o ensino quando contribui diretamente na redução das barreiras de tempo e espaço (MORAN, 1997). Dessa forma, o avanço da tecnologia mudou a forma de comunicação entre a sociedade e as novas gerações têm mostrado grande afinidade e gosto pelos recursos tecnológicos.

O uso de tecnologias no ensino superior teve uma revolução no modo de ensino aos estudantes, contribuindo de forma positiva no aprendizado, no entanto é preciso ter uma adaptação na maneira pedagógica de ensino (RIEDNER E PISCHETOLA, 2016). Ainda segundo os autores, a inserção da tecnologia valoriza um novo tipo de saber, o conhecimento intelectual.

Cabe ressaltar que esse conhecimento contribuirá para obter novas experiências, sempre buscando a tecnologia como um novo meio de aprendizado acadêmico (BATES, 2015).

Seguindo essa linha de raciocínio, entende-se que as mudanças ocasionadas pelo uso de tecnologias voltadas a educação propõe novas ferramentas no modo de aprender e ensinar, as quais disponibilizam meios de comunicação onde a sociedade acadêmica terá uma busca insaciável pelo aprendizado (BALADELI BARROS E ALTOÉ, 2012).

Salientando, o uso dos atuais meios de comunicação tendo como exemplo o Facebook, Whatsapp, Instagram, entre outros, restituem a interação e troca de conhecimentos que vai além da sala de aula, entre os professores e seus alunos e uma troca de conhecimentos geral no meio acadêmico (LEHMANN; PARREIRA, 2019).

Diante dos fatos mencionados, podemos destacar que a sociedade se baseia inteiramente em uma economia de conhecimento, portanto a educação tem um papel fundamental de desenvolver com os alunos essas técnicas digitais para que possam integrar esses novos conhecimentos as suas sociedades (HARGREAVES, 2004).

Como os smartphones estão popularizados e permitem fácil acesso a ferramentas computacionais, os dispositos moveis passam a apresentar um alto potencial para contribuir no processo de aprendizagem dos alunos (LEITE, 2014). Segundo Shuler (2009, p. 5) os “[...] dispositivos móveis podem ajudar a promover o conhecimento, as habilidades e perspectivas que as crianças precisarão para competir e cooperar no século 21 ”.

Dessa forma, a inserção dos recursos tecnológicos na educação apresentam potencial na mediação do conhecimento, propiciando novas oportunidades aos docentes ensinar e rompendo paradigmas, possibilitando aos alunos condições para aprender em um novo modelo de ensino mais autônomo e ativo (LOCATELLI ET AL.,2015).

Os autores Oliveira et al., (2016) apresentam que os dispositívos móveis e os aplicativos 
estão presentes na vida dos alunos e portanto, o docente precisa pensar no modo de utilizá-los nas aulas, de modo a contribuir no processo de ensino.

\section{Seleção das disciplinas do curso de Agronomia}

Para o desenvolvimento do aplicativo, foram selecionadas as disciplinas de Física, Bioquímica, Natureza e Propriedades dos Solos, Agrometeorologia e Comunicação e Extensão Rural.

A Física é uma disciplina complexa que exige do aluno conhecimento prévio do ensino médio, mas que, geralmente, não são abordadas adequadamente. Apesar disso, essa disciplina serve como base para a compreensão dos alunos em outras matérias importantes de atuação direta do agrônomo como mecanização agrícola e sistema de irrigação. A disciplina de Bioquímica é base para a disciplina de Melhoramento Genético, fundamental para que o agrônomo consiga realizar uma ótima atuação no mercado de trabalho. Normalmente essas disciplinas são ofertadas até o terceiro semestre do curso de Agronomia, de forma que os alunos iniciantes possam utilizar o aplicativo como forma de avaliar os conhecimentos adquiridos nas aulas.

Nas especificas, a disciplina de solos é de fundamental importância para a compreensão dos alunos, pois é a partir dela que o Agrônomo precisa realizar a correta adubação para que ocorra o crescimento adequado das plantas. A agrometeorologia é o ramo da meteorologia que estuda as relações de causa e efeito das condições meteorológicas com o meio rural e a produção agrícola. Já a disciplina de Comunicação e Extensão Rural apesar de não ser uma disciplina com conteúdo extenso, ela apresenta importância por conter itens de extensão, onde o aluno precisa aprender a comunicar e com os produtores, sejam eles de grandes fazendas ou pequenas propriedades.

\section{METODOLOGIA}

Para o desenvolvimento deste trabalho foi realizado inicialmente uma pesquisa teórica sobre as disciplinas dos semestres iniciais do curso de Agronomia, sendo utilizada como base o projeto pedagógico do curso de Agronomia da Universidade do Estado de Mato Grosso, campus de Nova Mutum. As disciplinas selecionadas para a primeira versão do aplicativo foram Física, Bioquímica, Natureza e Propriedades dos Solos, Agrometeorologia e Comunicação e Extensão Rural.

Para o desenvolvimento do aplicativo foi selecionado o sistema Android pois de acordo 
com Cardoso (2020) está presente em mais de 90\% dos celulares brasileiros.

Após a seleção de cinco disciplinas para fazerem parte do protótipo, foram elaboradas questões para compor um Quiz, sendo inicialmente cinco questões por disciplina. Também foi realizado um texto informativo com informações relevantes das disciplinas e conteúdos aborados nas questões do Quiz para que o usuário tivesse acesso para estudo futuro.

$\mathrm{Na}$ sequência, foi esquematizado o formato que as telas serão apresentadas para programação no programa AppInventor devido a simplicidade no processo de desenvolvimento por iniciantes em programação.

\section{RESULTADOS E DISCUSSÃO}

O aplicativo "Agro-Estudante" é um aplicativo criado em formato de Quiz para que os alunos dos primeiros semestres nos cursos de Agronomia possam estudar e conhecer um pouco do curso e das principais disciplinas que estudarão ao longo dos anos.

Para a primeira fase do protótipo, foram selecionadas cinco áreas sendo duas comuns a outros cursos, consideradas áreas gerais e três específicas do curso de Agronomia. As disciplinas das áreas gerais escolhidas são as disciplinas de Física e Bioquímica. $\mathrm{Na}$ área de disciplinas especificas optou-se, nesse primeiro momento, abordar as disciplinas de Natureza e Propriedades dos Solos, Agrometeorologia e Comunicação e Extensão Rural.

Nos subtópicos a seguir será apresentada uma breve descrição das telas e formatado de apresentação do aplicativo.

\section{Tela de abertura do aplicativo}

Ao acessar o aplicativo, será apresentada a tela de abertura (Figura 1) com um estudante carregando alguns livros, símbolo da Agronomia e o logo do Programa Uniciências da Universidade do Estado de Mato Grosso - Unemat. 


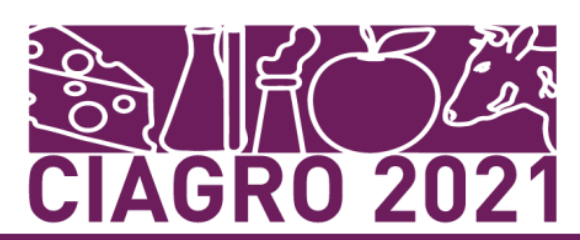

Figura 01: Tela de abertura do Aplicativo Agro estudante.

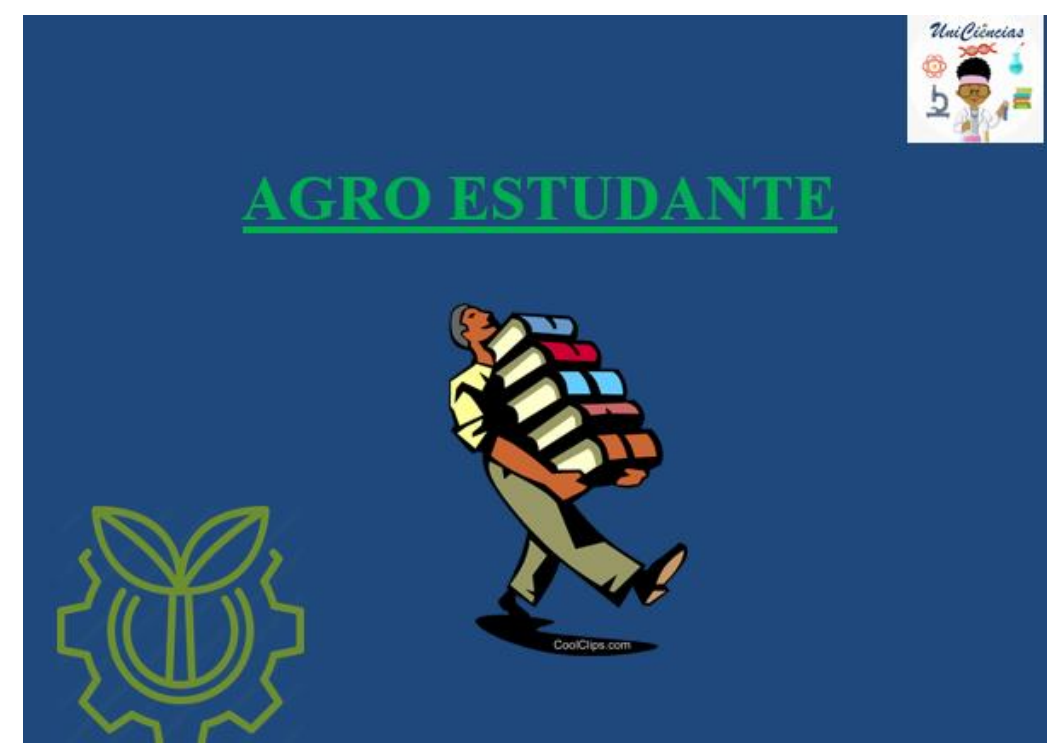

Fonte: Própria (2021).

A apresentação da tela de abertura demorará aproximadamente três segundos até seguir para a tela inicial.

\section{Tela de inicial do aplicativo}

Após abertura do aplicativo, o indivíduo será direcionado a tela inicial com o menu principal.

Nessa tela, o usuário poderá atribuir um nome ao avatar dele, e selecionar o avatar agroestudante. Nesse local será possível ainda selecionar uma roupa do guarda-roupa do avatar, onde alguns itens estarão bloqueados e só serão disponibilizados após o usuário terminar as missões do Quiz.

A Figura 2 apresenta os modelos iniciais de avatares que foram selecionados para a programação do protótipo. 
Figura 02: Avatares do aplicativo Agro estudante.
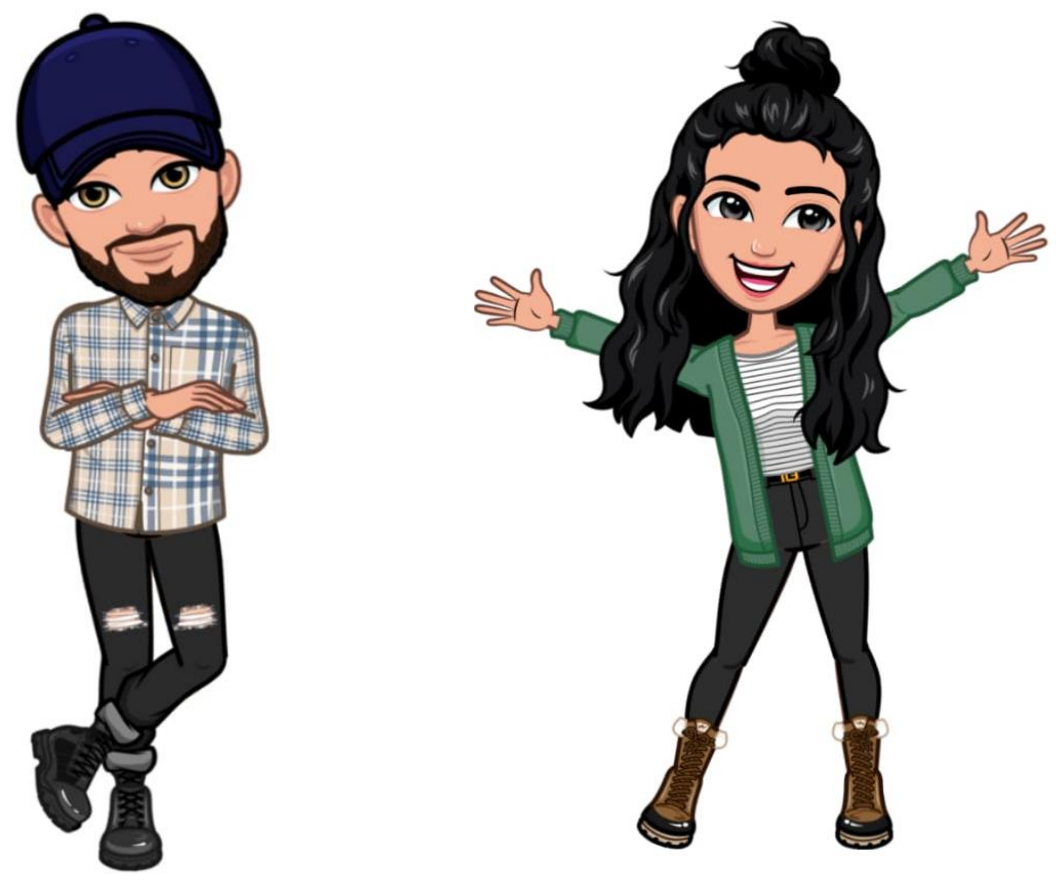

Fonte: Própria (2021).

Os avatares foram inspirados em dois bolsistas do grupo de pesquisa e desenhados no Programa Avatoon.

$\mathrm{Na}$ lateral da tela inicial, serão apresentados quatro menus que os usuários poderão acessar em qualquer uma das telas posteriores do aplicativo. Esse menu será fixo e irá conter acesso rápido ao apelido do avatar, a pontuação acumulada no Quiz e o guarda-roupa para modificar as roupas dos avatares (Figura 3).

Se o usuário clicar no apelido, será direcionado a tela inicial onde poderá alterar o apelido dado ao avatar. Se optar por entrar no guarda-roupa poderá ver as roupas ainda bloqueadas e se quiser, poderá alterar a vestimenta do avatar e terá acesso a pontuação até o momento. 


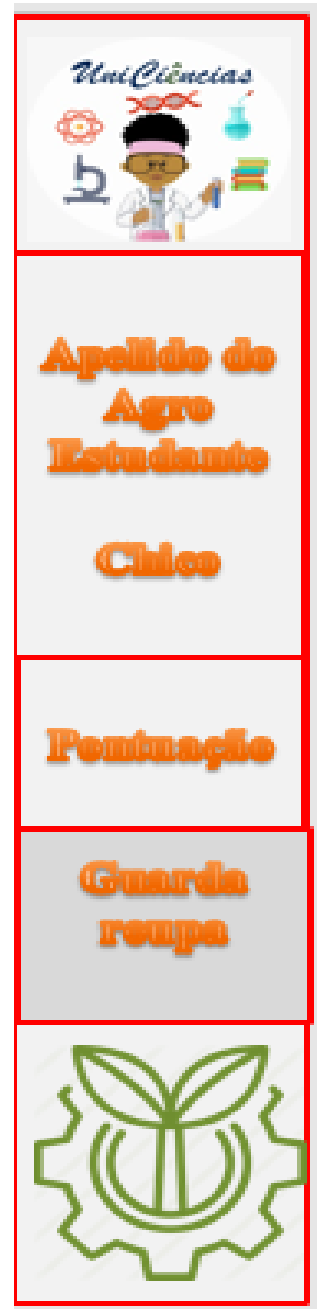

Fonte: Própria (2021).

Essa tela terá ainda a opção "iniciar" do jogo, onde o usuário será direcionado a tela seguinte com as opções das temáticas abordadas no aplicativo.

\section{Descrição das fases do jogo}

$\mathrm{Na}$ tela seguinte, o usuário será direcionado a tela onde está apresentada as fases seguintes que terá que percorrer no jogo, iniciando pelas questões da disciplina de física, bioquímica, natureza e propriedades do solo, agrometeorologia e por fim, comunicação e extensão rural. A Figura 4 apresenta o esboço da tela de fases. 


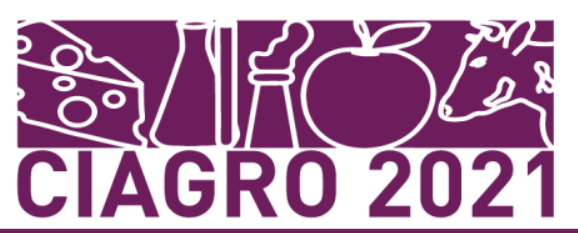

Figura 04: Menu das fases as telas do aplicativo

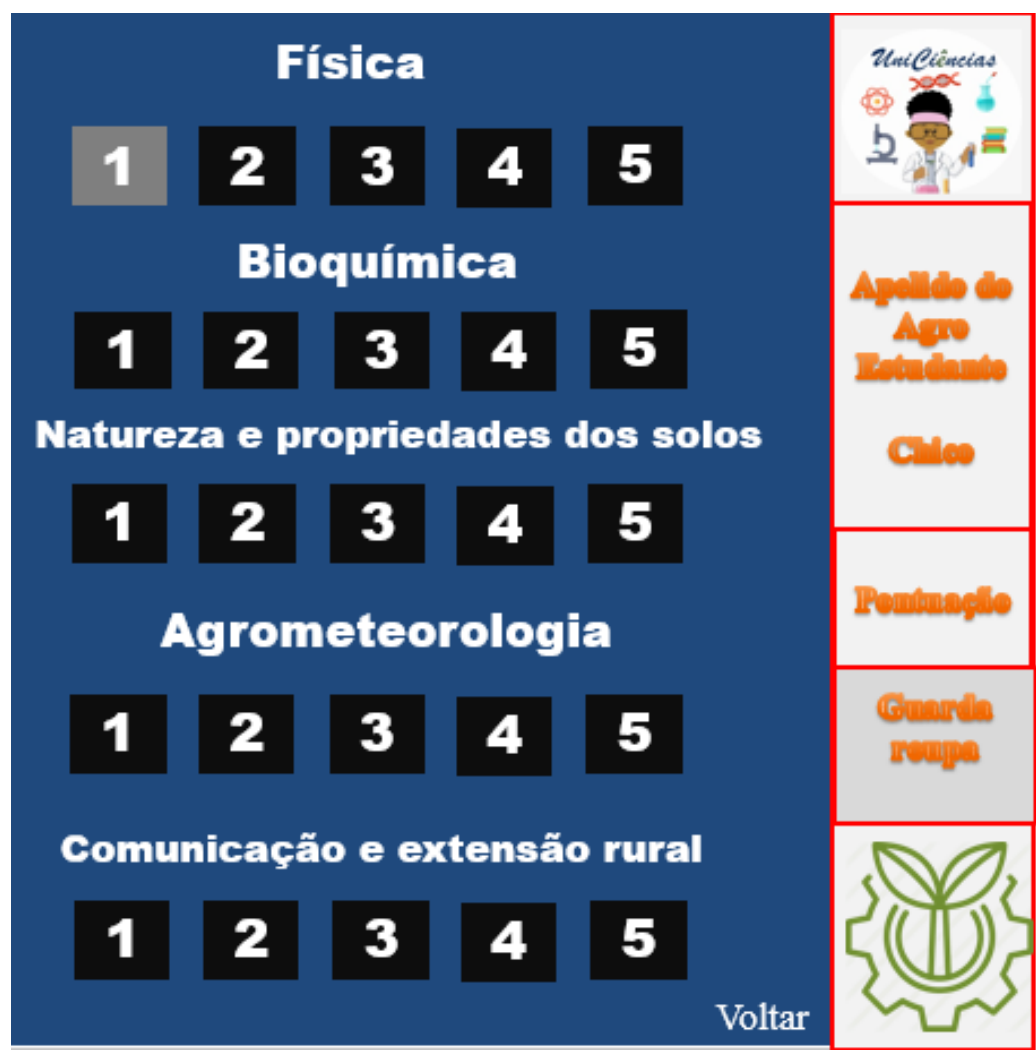

Fonte: Própria (2021).

Em cada uma das subfases será apresentada uma questão de múltipla escolha referente a fase selecionada com cinco alternativas. É importante ressaltar que o usuário somente terá acesso a subfase seguinte e respectivamente a próxima fase quando marcar corretamente a resposta.

Ao completar todas as questões da fase, o aluno será direcionado a uma tela onde poderá encontrar resumidamente informações importante sobre o conteúdo, de forma a fixar o aprendizado obtido durante a disciplina no curso de graduação. Nessa etapa ele terá a opção voltar ao menu inicial ou avançar para a fase seguinte.

A opção de apresentar parte do conteúdo resumidamente ao aluno tem por objetivo auxiliar no processo de aprendizagem das respectivas disciplinas.

\section{Descrição dos conteúdos selecionados para as fases do jogo}

Para seleção dos conteúdos de cada disciplina, foi priorizado aqueles que estão relacionados a outras disciplinas, como por exemplo bioquímica que é fundamental para melhoramento genético. Também foi selecionado conteúdos que o aluno precisa ter conhecimento pois serão aplicados diretamente na sua atuação profissional, como por exemplo, 
o conteúdo de assistência técnica na disciplina de Comunicação e Extensão Rural.

Na subfase de física, serão abordadas no aplicativo questões relacionadas aos cálculos de vazão para processo de irrigação, força de trabalho, potência de motores e cálculos de conversão simples como alqueires.

A subfase de bioquímica terá questões referente a assimilação do nitrogênio, processo de fotossíntese, importância dos nutrientes para as plantas e controle de ervas daninhas.

Na subfase de natureza e propriedade dos solos serão abordados conteúdos sobre formação das rochas, características dos solos para agricultura e composição do solo. Na subfase de agrometeorologia será abordado sobre declinação solar, elementos do clima e fatores climáticos e agricultura.

Na subfase de comunicação e extensão rural o usuário terá acesso a questões sobre assistência técnica, modelo e fases de extensão rural no Brasil.

As questões do protótipo serão retiradas de referências presentes nos Projetos Pedagógicos dos Cursos (PPC) de Agronomia e de provas do Exame Nacional de Desempenho dos Estudantes (ENADE) do curso de Agronomia.

\section{Pontuação}

Sempre que o usuário acertar a questão, aparecerá na tela uma mensagem de Parabéns! Se a resposta marcada for errada, a mensagem será de continue tentando.

Cada questão (subfases) terá inicialmente 50 pontos. Se o aluno errar uma vez, ela passará a valer 40 pontos até que tenha valor de 10 pontos quando esgotar as alternativas, ou seja, o aluno perde 10 pontos a cada resposta errada que marcar.

No final do jogo, o aluno poderá acumular um valor máximo de 1250 pontos e mínimo de 250 pontos. É importante destacar que a cada finalização das fases (disciplinas), o usuário terá acesso a outras roupas do avatar.

Sempre que errar a questão, o aluno será direcionado a seção de conteúdo da disciplina, para que possa rapidamente ler e fazer nova tentativa.

\section{Menu extra: Curso de Agronomia}

No final do aplicativo será apresentado um menu extra com informações sobre o curso de Agronomia.

Nesse menu, será abordado sobre a área de atuação do agrônomo, principais disciplinas que fazem parte do projeto pedagógico dos cursos de Agronomia além da importância do agrônomo para a agricultura. 


\section{Trabalhos existentes na literatura}

Em uma rápida busca de aplicativos que envolvam o curso de Agronomia no Play Store, foram encon5rados aplicativos que auxiliam o produtor, apresentem sobre o curso de Agronomia e simulados para concursos. Porém, não foi encontrado nenhum aplicativo voltado em exclusividade aos alunos ingressantes no curso e tenham itens que são desbloqueados conforme os acertos nas questões.

\section{CONCLUSÕES}

No presente trabalho foi desenvolvido um aplicativo em formato de perguntas e respostas que tem potencial para contribuir na aprendizagem de conteúdos relacionados a agronomia. O protótipo conta com cinco questões em cinco disciplinas diferentes, onde o usuário recebe pontuação para cada acerto e, em caso de erro, é encaminhado para uma seção de estudo sobre a área relacionada a questão.

Os conteúdos constantes no aplicativo são apresentados por avatares desenvolvidos com base nos bolsistas do programa Uniciências. Os conteúdos foram selecionados através do plano de curso de agronomia.

\section{REFERÊNCIAS}

BALADELI, A. P. D.; BARROS, M. S. F.; ALTOÉ, A. Desafios para o professor na sociedade da informação. Educar em Revista, Curitiba, n. 45, p. 155-165, set. 2012

BATES, Tony. Teaching in a digital age: guidelines for designing teaching and learning. Vancouver: Tony Bates Associates, 2015.

CARDOSO, Beatriz. 9 em cada 10 brasileiros usam celular Android, diz relatório do Google. Techtudo, 2020. Disponível em: <https://www.techtudo.com.br/noticias/2020/09/9-em-cada10-brasileiros-usam-celular-android-diz-relatorio-do-google.ghtml>. Acesso em: 04 nov. 2020.

CAROLINO, J. de A. Contribuições da Pedagogia de Projetos e do uso das Tecnologias de Informação e Comunicação (Tics) para o ensino de Geografia - um estudo de caso. 2007. Dissertação (Pós-Graduação em Educação) - Universidade de São Paulo, São Paulo, 2007.

CASTIONI, Remi et al . Universidades federais na pandemia da Covid-19: acesso discente à internet e ensino remoto emergencial. Ensaio: aval.pol.públ.Educ., Rio de Janeiro, v. 29, n. 111, p. 399-419, June 2021 . Available from <http://www.scielo.br/scielo.php?script=sci_arttext\&pid=S0104-

40362021000200006\&lng=en\&nrm=iso>. access on 13 Apr. 2021. Epub Feb 22, 2021. http://dx.doi.org/10.1590/s0104-40362021002903108. 
HARGREAVES, A. O ensino na sociedade do conhecimento: educação na era da insegurança. Porto Alegre: Artmed, 2004.

KENSKI, V. M. Educação e tecnologias: o novo ritmo da informação. 3. ed. São Paulo: Papirus, 2008.

KENSKI, Vani Moreira. Tecnologias e o ensino presencial e a distância. 9 ed. Campinas, SP: Papirus, 2010. pdf >.

LEHMANN, L.; PARREIRA, A. Instrumentos inovadores de aprendizagem: uma experiência com o WhatsApp Revista Lusófona de Educação, Lisboa, v. 43, n. 43, p. 75-89, maio 2019. Disponível em: https://revistas.ulusofona.pt/ index.php/rleducacao/article/view/6771. Acesso em: 4 jun. 2019.

LEITE, Bruno Silva. M-Learning: o uso de dispositivos móveis como ferramenta didática no Ensino de Química. Revista Brasileira de Informática na Educação, v. 22, n. 3, 2014.

LÉVY, P. Cibercultura. São Paulo: Editora 34, 1999.

LOCATELLI, Aline; ZOCH, Alana Neto; TRENTIN, Marco Antonio Sandini. TICs no Ensino de Química: Um Recorte do "Estado da Arte." Revista Tecnologias na Educação - Ano 7, n 12. Julho de 2015.

MORAES, Maria Cândida. Tecendo a rede, mas com que paradigma. Educação a distância: fundamentos e práticas. Campinas: UNICAMP/NIED, 2002.

MORAN, J. M. (1997). Como utilizar a internet na educação. Revista Ciência da Informação, 26(2), 146-153.

OLIVEIRA, Fábio Caires de; SOUTO, Daise Lago Pereira; CARVALHO, José Wilson P.; Seleção e análise de aplicativos com potencial para o ensino de química orgânica. Revista Tecnologias na Educação. n 8. v.17. Dez. 2016.

PISCHETOLA, M. (2016). Inclusão digital e educação: a nova cultura da sala de aula. Petrópolis/Rio de Janeiro: Vozes/PUC-Rio.

RIEDNER, D. T., D.; PISCHETOLA, M. Tecnologias Digitais no Ensino Superior: uma possibilidade de inovação das práticas?. Educação, Formação \& Tecnologias - ISSN 1646933X, América do Norte, 9, dez. 2016. Disponível em: <https://eft.educom.pt/index.php/eft/article/view/526>

ROVAI, A. P.; JORDAN, H. Blended learning and sense of community: a comparative analysis with traditional and fully online graduate courses. The International Review of Research in Open and Distributed Learning, [s. 1.], v. 5, n. 2, p. 1-13, Aug. 2004. https://doi.org/10.19173/irrodl.v5i2.192

SHULER, C. Pockets of potential Using Mobile Technologies to Promote Children's Learning, Ed.M. January 2009. 
TEODORO, A.; VASCONCELOS, M. L. Ensinar e aprender no ensino superior: por uma epistemologia da curiosidade na formação universitária. São Paulo: Cortez/Mackienze, 2005.

VALENTE, J. A. (Org.). O computador na sociedade do conhecimento. Campinas: NIED/UNICAMP, 1999.

WILEY, David A. et al. Conectando objetos de aprendizagem à teoria do design instrucional: Uma definição, uma metáfora e uma taxonomia. O uso instrucional de objetos de aprendizagem , v. 2830, n. 435, p. 1-35, 2000. 